\title{
Perfil dos medicamentos prescritos para idosos em uma Unidade Básica de Saúde do município de liúi-RS
}

\author{
Prescription medicine profile to elderly in a Basic Health Unit of the city of liui, Brazil
}

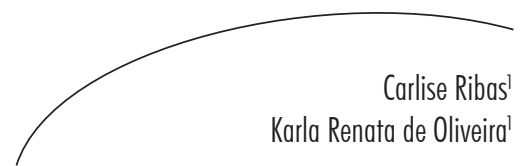

\section{Resumo}

Buscou-se identificar os medicamentos utilizados pelos idosos atendidos numa Unidade Básica de Saúde do município de Ijuí-RS e entre estes, os medicamentos potencialmente inapropriados para esta faixa etária, além de verificar as potenciais interações envolvendo esses medicamentos. Realizou-se estudo transversal e documental, a partir de 429 prescrições aviadas de janeiro a novembro de 2010, para 286 idosos. A maioria dos idosos era formada por mulheres $(67,13 \%$ ) e a idade média foi de $71,59 \pm 7,77$ anos; prevaleceu a faixa etária de 60 a 79 anos. Foram dispensadas 87 especialidades farmacêuticas distintas,

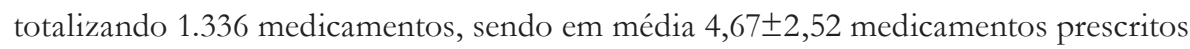
por idoso, variando de um a 15 medicamentos/idoso. Verificou-se polifarmácia em 135 idosos (47,20\%). Os medicamentos mais prescritos atuam no sistema cardiovascular, no aparelho digestivo e metabolismo e no sangue e órgão hematopoiéticos. Do total de especialidades farmacêuticas, 14 (16,09\%) foram considerados medicamentos potencialmente inapropriados, de acordo com os critérios de Beers, que foram prescritos a $62(21,68 \%)$ idosos. Estão expostos a interações medicamentosas 40 (56,34\%) idosos que receberam medicamentos potencialmente inapropriados, dos quais $27(67,50 \%)$ estão expostos a duas ou mais interações. Verificaram-se polimedicação e uso de medicamentos potencialmente inapropriados entre os idosos em estudo, mas estas podem estar sendo necessárias, pois foram identificados medicamentos indicados para as doenças crônicas prevalentes nessa faixa etária. Diante disso, sugere-se o uso dos critérios de Beers na avaliação da farmacoterapia em idosos, destacando-se ainda a necessidade de incorporar o uso de terapias não farmacológicas, a fim de favorecer a redução no consumo de medicamentos.

\section{Abstract}

This study aimed to identify the drugs used by elderly patients treated in a Basic Health Unit (UBS) in the city of Ijuí, state of Rio Grande do Sul, Brazil, identify the potentially inappropriate medications for this age group, and assess potential interactions between these drugs. It is a cross-sectional and documental study on 429

Curso de Farmácia, Departamento de Ciências da Vida. Universidade Regional do Noroeste do Estado do Rio Grande do Sul. Ijuí, RS, Brasil.

Palavras-chave:

Idosos. Interações de Medicamentos. Prescrições de Medicamentos. Uso de Medicamentos. Medicamentos Farmacoterapia. 
prescriptions dispensed from January to November 2010, for 286 elderly. Most elderly are female $(67.13 \%)$, mean age $71.59 \pm 7.77$ years, most of them aged $60-79$ years. Were dispensed 87 different pharmaceuticals, totaling 1,336 medications, with mean of $4.67 \pm 2.52 \mathrm{drugs} / \mathrm{elderly} /$ prescription, ranging from 1 to $15 \mathrm{drugs} / \mathrm{elderly}$. There was polypharmacy in $135(47.20 \%)$ elderly. The most commonly prescribed drugs act on the cardiovascular system, digestive system, metabolism, blood and organs. Of the total number of pharmaceutical specialties, 14 (16.09\%) were considered potentially inappropriate medications according to the Beers criteria, which were prescribed to $62(21.68 \%)$ elderly. Forty elderly $(56.34 \%)$ are exposed to potentially inappropriate drug interactions, among these $27(67.50 \%)$ are exposed to two or more interactions. Polypharmacy and potentially inappropriate medications were identified in this study, but these can be necessary to the elderly considering the prevalence of chronic diseases in this age group. So we suggest the use of Beers criteria in the evaluation of pharmacotherapy to the elderly, emphasizing the need to incorporate the use of nonpharmacological therapies so as to encourage reduction to the medicine use.
Key words: Elderly. Drug Interactions. Drug Prescriptions. Drug Utilization. Drug Therapy. Pharmacotherapy.

\section{INTRODUÇÃO}

Segundo o Ministério da Saúde (MS), cerca de 17,6 milhões de brasileiros são idosos, e a doença e os medicamentos estão presentes no cotidiano desta população, sendo muito particulares as alternativas para gerenciar tal situação. ${ }^{1}$ Neste sentido, os medicamentos representam um dos itens mais importantes da atenção à saúde do idoso, uma vez que esta faixa etária tende a usar mais produtos farmacêuticos e apresenta particularidades farmacocinéticas e farmacodinâmicas que se combinam com os múltiplos processos patológicos, influências ambientais e variações genéticas, o que torna os idosos particularmente vulneráveis a efeitos adversos. ${ }^{2,3}$

Com relação ao uso de medicamentos em idosos, destaca-se a prática da polifarmácia, ${ }^{4}$ que, somada à presença de várias doenças, alterações fisiológicas e atendimento por diversos médicos, torna os idosos o principal grupo de risco para utilizar medicamentos potencialmente inapropriados (MPI), além de provocar interações medicamentosas e reações adversas a esses medicamentos. ${ }^{5}$

Orientações adequadas sobre a correta utilização dos medicamentos, dosagens e intervalos, fornecidas aos idosos e seus familiares, são essenciais à manutenção da qualidade de vida do idoso. ${ }^{1}$ Diante disso, foram estabelecidos critérios para avaliar a qualidade da utilização de medicamentos entre os idosos, dentre os quais se destacam os critérios de Beers, ${ }^{6}$ atualizados por Fick et al. ${ }^{7}$ Este, segundo Quinalha \& Correr, $^{8}$ é o método mais utilizado para avaliar os efeitos dos medicamentos prescritos aos idosos e auxiliar na identificação de MPI prescritos para os pacientes geriátricos.

A avaliação da farmacoterapia em idosos, portanto, é um importante instrumento de avaliação da qualidade da atenção prestada a este grupo etário, sendo que esforços para aprimorar a seleção, a prescrição, a dispensação e a utilização de fármacos devem constituir prioridade nos programas de atenção ao idoso. ${ }^{9}$ Com vistas a contribuir para a qualificação do serviço de saúde do município no que se refere à atenção ao idoso, a gestão de recursos destinados a aquisição de medicamentos e organização da assistência farmacêutica no município, buscou-se estudar os medicamentos dispensados a idosos em uma Unidade Básica de Saúde (UBS) de Ijuí-RS.

O objetivo deste estudo foi identificar os medicamentos utilizados pelos idosos atendidos em uma UBS do município de Ijuí-RS e, entre estes, os medicamentos potencialmente inapropriados (MPI) prescritos, além de verificar as potenciais interações envolvendo MPI. 


\section{METODOLOGIA}

Trata-se de um estudo transversal, baseado na análise documental de prescrições. A coleta de dados foi realizada em duas etapas: inicialmente, buscou-se o cadastro dos usuários da UBS, com o objetivo de identificar os usuários com idade igual ou superior a 60 anos. A segunda etapa foi realizada com base nas prescrições aviadas a estes usuários no local do estudo, no período de janeiro a novembro de 2010. Assim, a amostra foi constituída pelos idosos cadastrados na UBS estudada, que receberam prescrição de medicamentos, aviadas de janeiro a novembro de 2010. Foi utilizado um instrumento de coleta de dados, o qual permitiu, a partir do cadastro e das prescrições, obter informações referentes a idade, sexo e medicamentos utilizados pelos idosos.

Os medicamentos identificados foram classificados de acordo com a Classificação Anatômica Terapêutica Química ${ }^{10}$ (Anatomical Therapentic Chemical - ATC, do Nordic Council on Medicines, Index 2011) e, utilizando os critérios de Beers, ${ }^{7}$ foi possível identificar os MPI prescritos para os idosos. Foram considerados medicamentos de uso contínuo aqueles prescritos por um período igual ou superior a 30 dias, e esporádicos os que foram prescritos por tempo determinado, inferior a 30 dias. Foram analisados dados como polifarmácia, que, segundo Souza, Santos \& Silveira, ${ }^{4}$ é o uso de pelo menos um medicamento desnecessário ou de cinco ou mais fármacos em associação, e interações medicamentosas, classificadas de acordo com Brasil $^{11}$ e Baxter. ${ }^{12}$

Soares et al..$^{13}$ destacam que os critérios de Beers $^{7}$ são constituídos por dois conjuntos de medicamentos, um incluindo substâncias que devem ser evitadas nos pacientes idosos e o outro apresentando situações clínicas e medicamentos que devem ser evitados na sua presença. Neste estudo, os medicamentos foram classificados como MPI independentemente de serem inapropriados para os idosos em geral ou em situações específicas, tendo em vista identificar na população estudada o maior número de medicamentos inapropriados para essa faixa etária.

Para o processamento dos dados, foi utilizado o programa Statistical Package for Social Science - SPSS (versão 18.0, Chigago-IL, EUA). Na análise estatística, as variáveis categóricas foram analisadas por meio do teste qui-quadrado de Pearson e/ou teste exato de Fisher. Os resultados foram apresentados em frequência absoluta e relativa. Todos os testes foram aplicados utilizando-se intervalo de confiança (IC) de 95\%, considerando-se $\mathrm{p}<0,05$ como nível mínimo de significância estatística.

A pesquisa foi aprovada pelo Comitê de Ética em Pesquisa da Universidade Regional do Noroeste do Estado do Rio Grande do Sul - CEP/Unijuí, sob o parecer consubstanciado n ${ }^{\circ}$ 081/2011

\section{RESULTADOS}

Foram identificados no cadastro da UBS 297 idosos, dos quais 286 receberam medicamentos no período do estudo, para os quais foram aviadas 429 prescrições. Dos idosos atendidos na UBS, 3,7\% (11) não utilizavam medicamentos no período do estudo, por isso foram excluídos do estudo.

A idade média dos idosos que constituíram a amostra foi $71,59 \pm 7,77$ anos, sendo a idade mínima 60 e a máxima 93 anos. Com relação ao sexo, a maioria $(67,13 \%)$ era do sexo feminino. Com relação à faixa etária, verificou-se maior número de idosos com idades entre 60 e 79 anos (tabela 1). 
Tabela 1. Distribuição dos idosos de acordo com sexo e faixa etária. Ijuí-RS, 2010.

\begin{tabular}{l|cc|cc}
\hline & \multicolumn{2}{|c|}{ Sexo } & $\mathrm{n}$ & \multicolumn{2}{c}{ Masculino } \\
Faixa etária & $\mathrm{n}$ & $\%$ & $\mathrm{n}$ & $\%$ \\
\hline 60-69 anos & 78 & 40,63 & 45 & 47,87 \\
$70-79$ anos & 73 & 38,02 & 39 & 41,49 \\
$80-89$ anos & 37 & 19,27 & 8 & 8,51 \\
$90-99$ anos & 4 & 2,02 & 2 & 2,13 \\
\hline Total & 192 & 100,00 & 94 & 100,00 \\
\hline
\end{tabular}

A partir das 429 prescrições aviadas, foram prescritas 87 especialidades farmacêuticas distintas, totalizando 1.336 medicamentos. Em média foram prescritos $4,67( \pm 2,52)$ medicamentos por idoso, variando de um a 15 medicamentos por pessoa, sendo que as mulheres tiveram até 15 medicamentos prescritos/idoso e os homens, até dez. Dos 286 idosos, 47,20\% (135) receberam mais de cinco medicamentos concomitantemente e 5,18\% (20) fizeram uso de monoterapia.

A tabela 2 apresenta a distribuição dos idosos por sexo de acordo com os medicamentos prescritos segundo a classificação ATC primeiro nível, onde se observa maior número de medicamentos prescritos às mulheres, e que mais de $90 \%$ dos medicamentos prescritos tanto para homens quanto para mulheres atuam no sistema cardiovascular.

Os medicamentos mais prescritos atuam no sistema cardiovascular (tabela 3), prevalecendo enalapril (30,54\%) e hidroclorotiazida (28,90\%), seguidos pelos que atuam no aparelho digestivo e metabolismo, e no sangue e órgãos hematopoieticos.

A análise estatística mostra relação entre os gêneros quanto à prescrição de medicamentos do grupo anatômico "B sangue e órgãos hematopoieticos" $(\mathrm{p}<0,001)$ e quanto à prescrição do grupo "N sistema nervoso" $(\mathrm{p}<0,001)$. $\mathrm{Na}$ população estudada, ambos os medicamentos foram mais prescritos ao sexo feminino. 


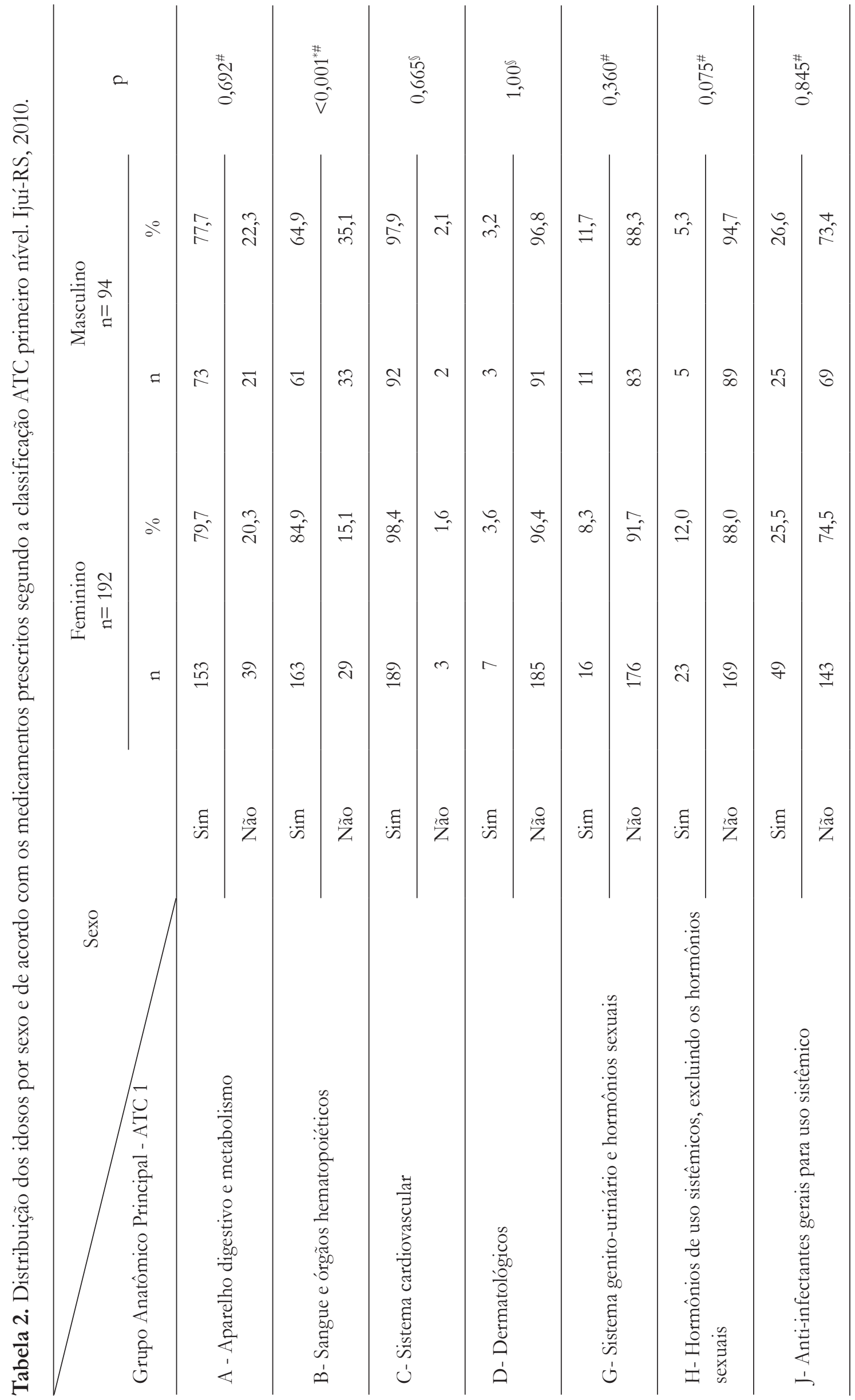




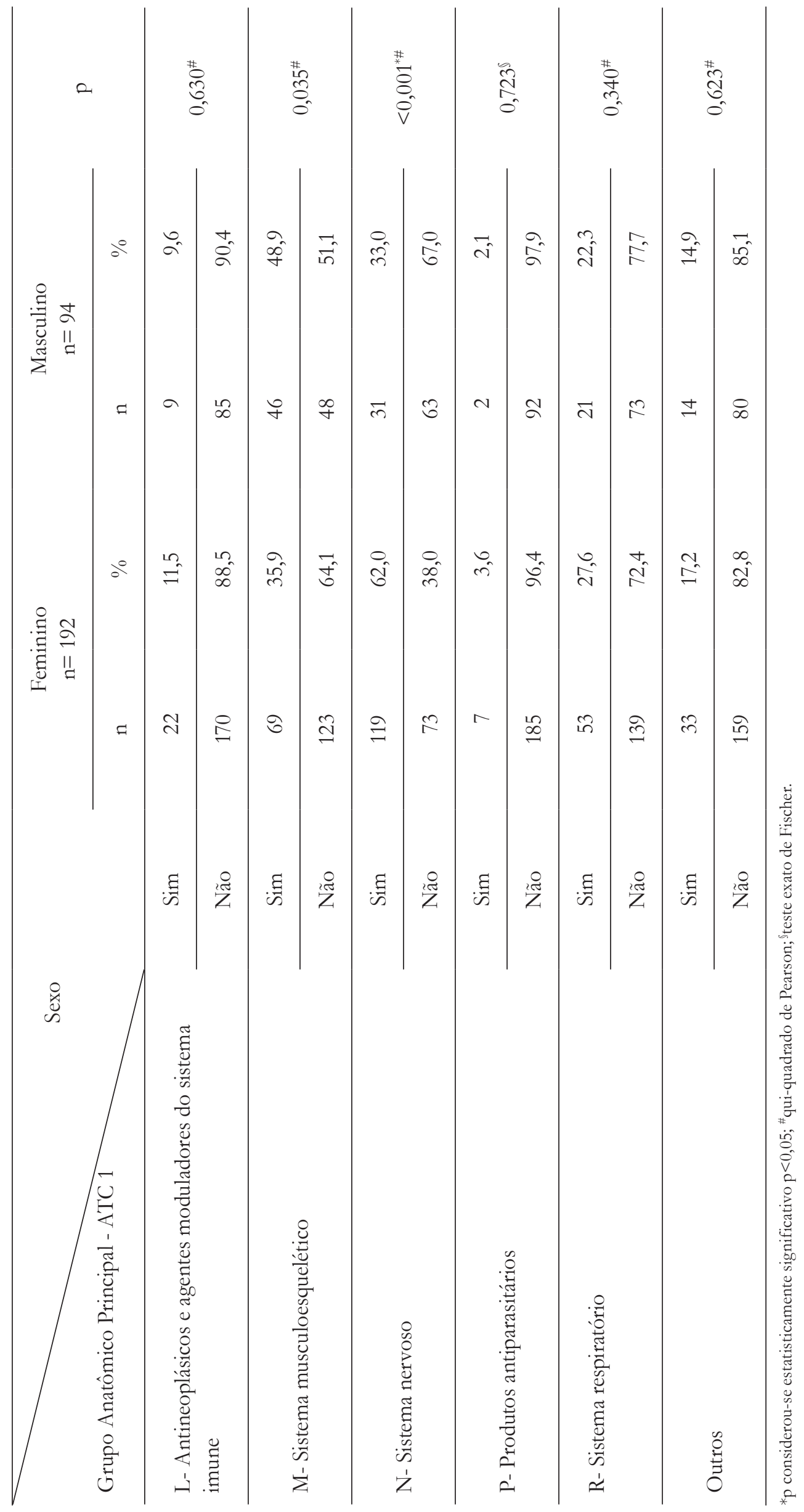




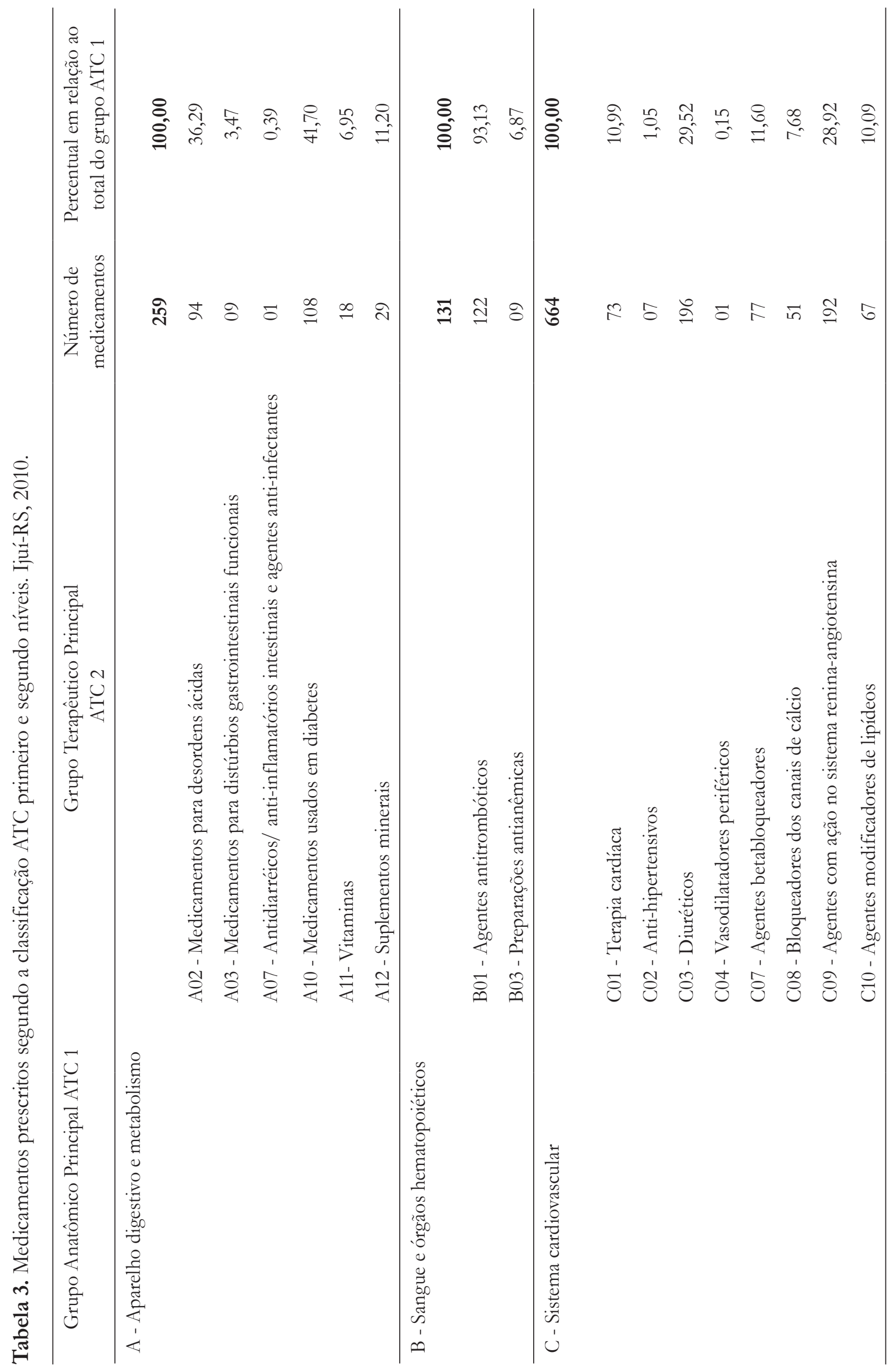




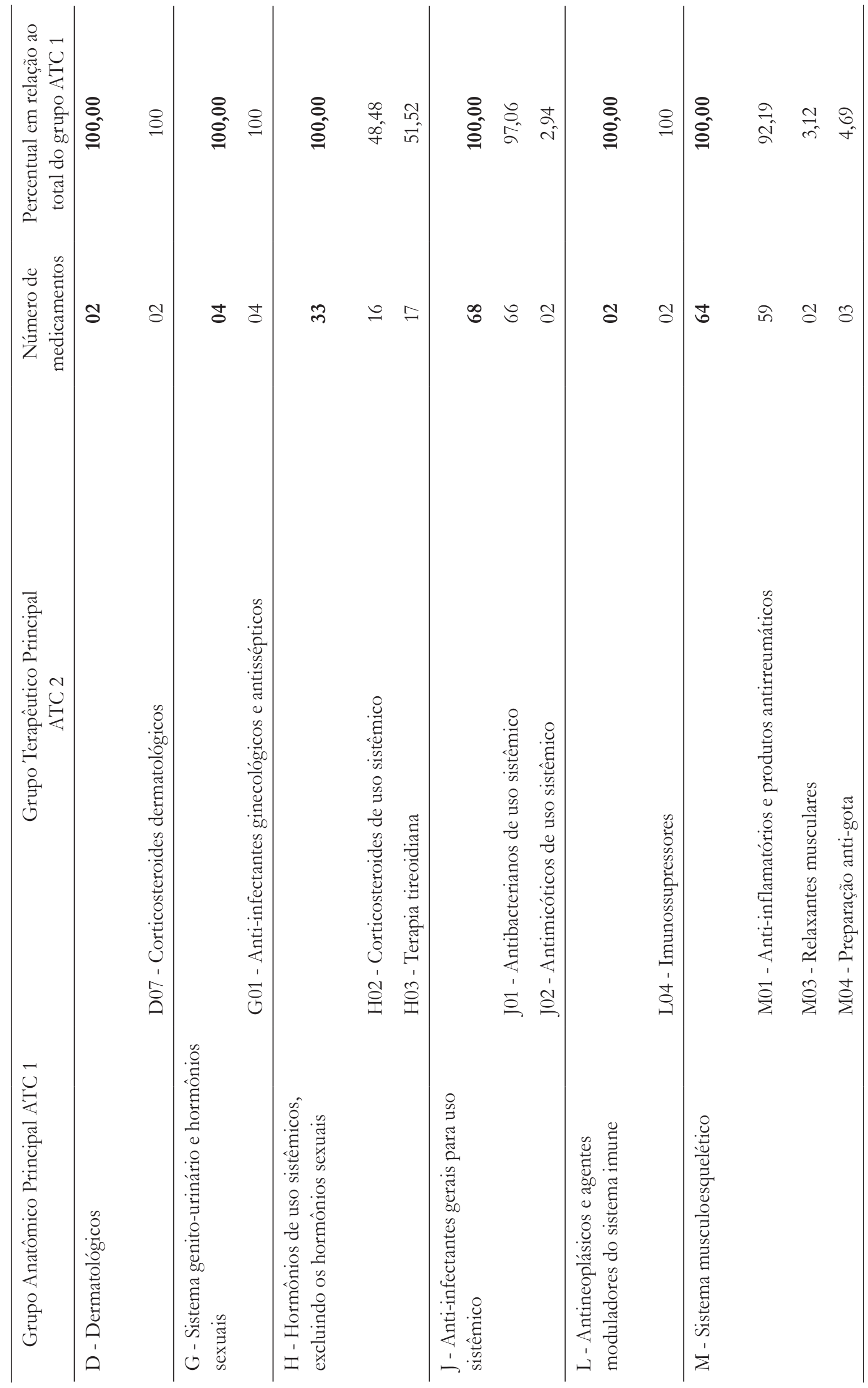


Prescrição de medicamentos para idosos

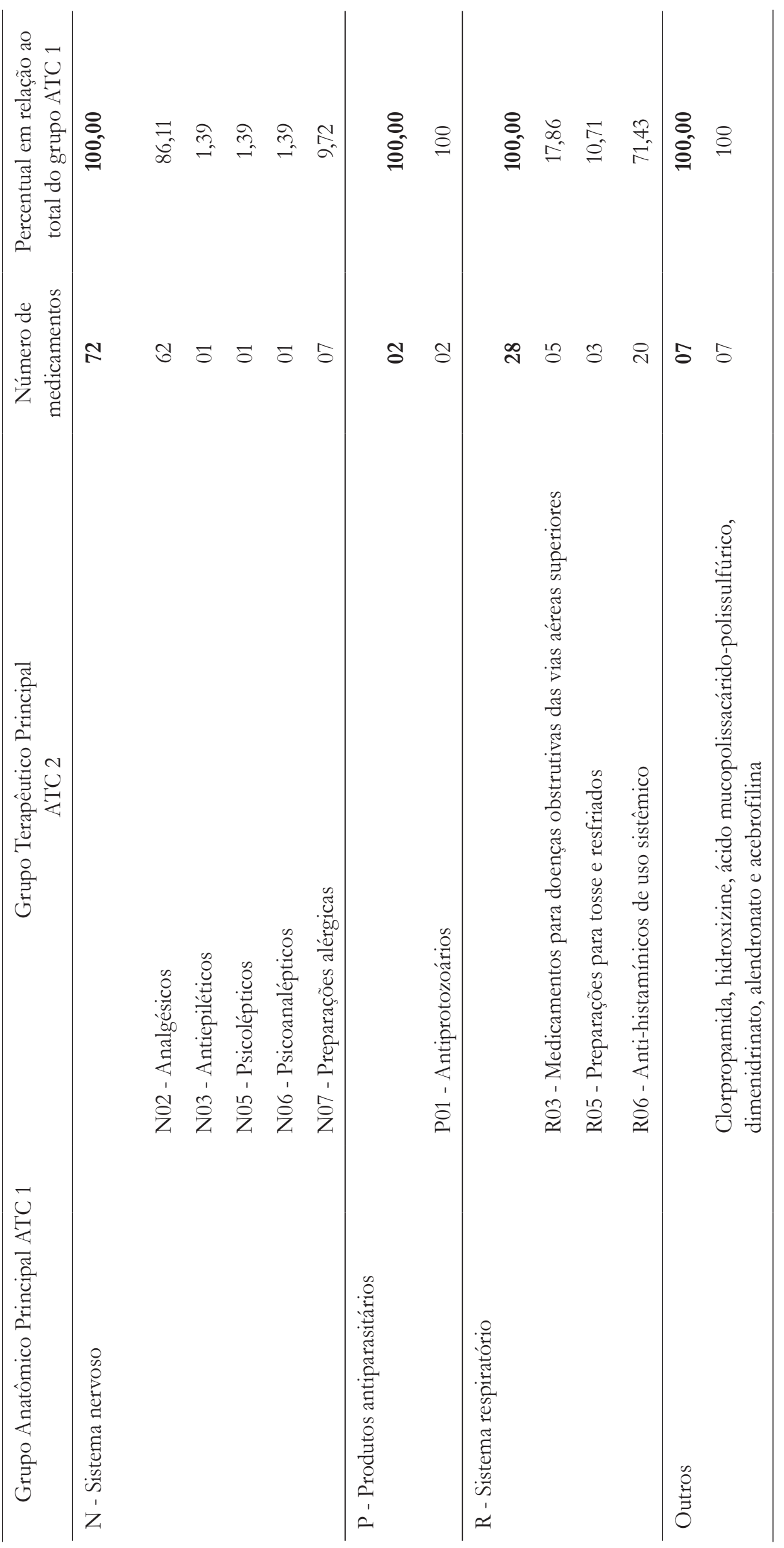


Dentre os medicamentos prescritos aos idosos, 66,67\% (58) são de uso contínuo e 33,33\% (29) foram prescritos de forma esporádica. Deste total, foram identificados 16,09\% (14) MPI, os quais foram prescritos para 62 idosos, que representam $21,68 \%$ da amostra analisada; destes indivíduos, 85,48\% (53) utilizaram um, e nove $(14,52 \%)$ usaram dois MPI. Destaca-se que outros nove idosos $(3,15 \%)$ receberam prescrição contendo digoxina, mas em dosagem menor que a considerada inapropriada por Fick et al. ${ }^{7}$

Os MPI prescritos para a população estudada estão apresentados na tabela 4.

Tabela 4. Medicamentos potencialmente inapropriados para idosos prescritos para os idosos estudados. Ijuí-RS, 2010.

\begin{tabular}{|c|c|c|}
\hline Medicamento Potencialmente Inapropriado & $\mathrm{n}=14$ & $\%=16,09$ \\
\hline Digoxina $^{2}$ & 27 & 40,30 \\
\hline Amiodarona & 15 & 22,39 \\
\hline Nifedipino & 5 & 7,46 \\
\hline Metoclopramida ${ }^{1}$ & 4 & 5,97 \\
\hline Sulfato ferroso & 3 & 4,48 \\
\hline Clorzoxazona & 3 & 4,48 \\
\hline Metildopa & 2 & 2,99 \\
\hline Ciclobenzaprina & 2 & 2,99 \\
\hline Clopidogrel $^{1}$ & 1 & 1,49 \\
\hline Clorpropramida & 1 & 1,49 \\
\hline Amitriptilina $^{1}$ & 1 & 1,49 \\
\hline Lorazepam & 1 & 1,49 \\
\hline Clonidina & 1 & 1,49 \\
\hline Hidroxizine & 1 & 1,49 \\
\hline
\end{tabular}

${ }^{1}$ Medicamentos inapropriados em algumas situações patológicas; ${ }^{2}$ Medicamento inapropriado quando em doses superiores que $0,125 \mathrm{mg} / \mathrm{dia}$.

Analisando os $14 \mathrm{MPI}$, verificou-se que três $(21,43 \%)$ estão envolvidos em nove potenciais interações medicamentosas distintas entre si, totalizando 77 interações (tabela 5). Considerando o total de usuários que receberam MPI (71), incluindo os que receberam digoxina em dosagem inferior à inapropriada, 56,34\% (40) idosos estão expostos a interações medicamentosas identificadas, sendo que $67,50 \%$ (27) deles estão expostos a duas ou mais interações. 
Tabela 5. Frequência de interações medicamentosas envolvendo MPI, conforme classificação ATC primeiro nível. Ijuí-RS, 2010.

\begin{tabular}{lccc}
\hline $\begin{array}{l}\text { Classes terapêuticas envolvidas em interações } \\
\text { medicamentosas conforme a classificação ATC }\end{array}$ & Interações medicamentosas & $\mathrm{n}=77$ & $\%$ \\
\hline & Digoxina + Espironolactona & 23 & 29,87 \\
& Digoxina + Furosemida & 19 & 24,67 \\
& Digoxina + Captopril & 9 & 11,69 \\
& Digoxina + Hidroclorotiazida & 6 & 7,79 \\
$\begin{array}{l}\text { Sistema cardiovascular } \\
\text { n=63 (29,71\%) }\end{array}$ & Digoxina + Amiodarona & 6 & 7,79 \\
& Nifedipino + Atenolol & 1 & 1,30 \\
& Nifedipino + Propranolol & 1 & 1,30 \\
\hline $\begin{array}{l}\text { Sistema cardiovascular }+ \text { Sistema } \\
\text { musculoesquelético }\end{array}$ & Digoxina + AINEs & 10 & 12,99 \\
\hline $\begin{array}{l}\text { Sistema cardiovascular }+ \text { Aparelho digestivo } \\
\text { e metabolismo }\end{array}$ & Nifedipino + antidiabéticos & 2 & 2,60 \\
\hline
\end{tabular}

\section{DISCUSSÃO}

A senescência é um processo natural de diminuição progressiva da reserva funcional dos indivíduos que em condições normais não causa problemas de saúde; diferencia-se do processo de senilidade, que ocorre em condições de sobrecarga, podendo ocasionar uma condição patológica que requeira assistência ${ }^{1}$. Por ser o grupo etário que cresce mais rapidamente no Brasil, a demanda dos idosos por recursos de saúde é intensa, tanto no que se refere à utilização dos serviços, quanto no que diz respeito ao uso de medicamentos. ${ }^{9}$ No presente estudo, a prevalência da faixa etária 60-79 anos, independentemente do sexo, assemelha-se aos resultados obtidos por outros estudos realizados em Minas Gerais ${ }^{14}$ e São Paulo, ${ }^{15-17}$ envolvendo idosos hospitalizados ${ }^{16,17}$ e usuários do sistema público de saúde. ${ }^{14,15}$
Com relação ao maior número de medicamentos prescritos às mulheres em relação aos homens, observado neste estudo, Rozenfeld ${ }^{18}$ destaca num estudo de revisão que isso pode estar relacionado ao fato de elas apresentarem pior estado funcional e de saúde autorreferida, e maior número de sintomas depressivos e hospitalizações, necessitando, portanto, de maior número de medicamentos para controle sintomático dessas doenças.

Pandolfi, Piazzolla \& Louzada, ${ }^{19}$ em estudo realizado numa instituição de longa permanência do Distrito Federal, observaram que os homens não utilizavam mais que sete fármacos, o que difere do encontrado neste estudo. Destacam-se, nesse contexto, a elevada prescrição e utilização de medicamentos pelos idosos atendidos no local do estudo, superiores ao verificado em instituições de longa permanência ${ }^{19}$ e instituições hospitalares. ${ }^{17}$ 
Simões \& Marques $^{20}$ destacam o consumo cada vez maior de medicamentos pelos idosos e apontam que esse é o grupo etário que mais utiliza medicamentos na sociedade, representando cerca de 50\% dos multiusuários, o que se justifica pelo aumento da prevalência de doenças crônicas com a idade.

Com relação à polifarmácia, os resultados obtidos no presente estudo assemelham-se aos encontrados por Lucchetti et al., ${ }^{16}$ que avaliaram idosos hospitalizados e observaram que 46,4\% faziam uso de mais de cinco medicamentos concomitantemente. Cabe salientar a elevada taxa de polifarmácia nas prescrições analisadas, já que Araújo, Magalhães \& Chaimowicz, ${ }^{21}$ que avaliaram a utilização de medicamentos por idosos no serviço público de saúde de Belo Horizonte-MG, verificaram o uso de cinco ou mais medicamentos em cerca de $25 \%$ dos idosos.

No que se refere aos medicamentos prescritos, considerando a classificação ATC, a maioria dos idosos analisados neste estudo recebeu medicamentos que atuam no aparelho cardiovascular, seguidos pelos que atuam no aparelho digestivo e metabolismo e os que atuam no sangue e órgãos hematopoiéticos (tabela 3). Esse resultado é similar ao encontrado por Acurcio et al. ${ }^{22}$ em estudo realizado com idosos não hospitalizados de Belo HorizonteMG, que verificou prevalência do uso de diuréticos, fármacos que atuam no sistema renina-angiotensina e medicamentos usados no tratamento do diabetes mellitus.

Conforme o nível 2 da classificação ATC (tabela 3), os diuréticos, seguidos pelos agentes com ação no sistema renina-angiotensina, agentes antitrombóticos e hipoglicemiantes, estão entre os mais prescritos, o que reforça a prevalência de doenças cardiovasculares, bem como diabetes mellitus na população estudada. Esses dados refletem a realidade brasileira no que se refere às doenças prevelentes ${ }^{23-25}$ e os medicamentos prescritos a idosos identificados em estudos realizados com esse grupo etário em diferentes estados brasileiros. . $719,26,27^{2}$
Frequentemente, os idosos estão expostos à polifarmácia devido à prevalência de doenças crônico-degenerativas e várias outras comorbidades. ${ }^{19,25,28,29}$ Por esta razão, Rozenfeld ${ }^{18}$ não considera esta prática sinônimo de prescrição inapropriada. A situação descrita corrobora o que preconiza o Ministério da Saúde com relação aos medicamentos para o controle das doenças cardiovasculares. ${ }^{28}$ Sugere a associação de medicamentos considerando que a monoterapia inicial é eficaz em cerca de $40-50 \%$ dos casos e que, para a maioria, com o transcurso do tempo é necessário adotar terapias combinadas, justificando a exposição dos idosos estudados a múltiplos fármacos. ${ }^{28}$

Assim, a polifarmácia, que é uma prática clínica comum nos idosos, pode constituir um tratamento personalizado, desde que o médico prescreva exclusivamente medicamentos necessários para a doença em questão. Permite assim identificar efeitos colaterais e minimizar os custos do tratamento, ou seja, acompanhar e orientar os usuários de vários medicamentos é fundamental, para que a polifarmácia não constitua fator de risco para o agravo da saúde do idoso., ${ }^{1,20,21}$

Por outro lado, surge a preocupação com a prescrição inapropriada, que ocorre quando os riscos superam os possíveis benefícios da farmacoterapia ou as doses e/ou frequência de uso são superiores as recomendadas. ${ }^{7,30}$ Portanto, os efeitos prejudiciais do uso de medicamentos por idosos impulsionam os prescritores, farmacêuticos e pesquisadores a desenvolver e aplicar instrumentos para facilitar a identificação de MPI. ${ }^{9}$

Neste estudo, verificou-se que uma parcela dos medicamentos prescritos aos idosos é de alguma forma inapropriada para esta faixa etária, resultado superior ao encontrado por Araújo, Magalhães \& Chaimowicz. ${ }^{21}$ Destaca-se o uso de uma substância que pode causar toxicidade devido à redução na sua depuração renal e outra que, além de apresentar baixa eficácia em pacientes geriátricos, está envolvida em inúmeras interações medicamentosas. ${ }^{7}$ 
Os critérios de Beers ${ }^{7}$ apresentam ainda alguns medicamentos que devem ser evitados pelos idosos em determinadas doenças ou condições, dentre os quais se destacam metoclopramida, clopidogrel e amitriptilina, que constavam em algumas prescrições analisadas neste estudo (tabela 4).

Farfel et al. ${ }^{30}$ observaram em seu estudo que mais de um terço dos atendimentos de emergência, relacionados a eventos adversos em adultos com mais idade, foram causados por fármacos listados nos critérios de Beers. ${ }^{7}$ Os autores ainda chamam a atenção para a prescrição inapropriada em idosos, a qual resulta frequentemente em atendimentos de baixa complexidade na emergência, mas algumas vezes pode ocasionar lesões graves ou irreversíveis.

Rozenfeld ${ }^{18}$ enfatiza que a proporção de usuários de MPI é um importante indicador de qualidade da assistência médico-sanitária, sendo útil para avaliar "casas de repouso", clínicas geriátricas e assistência ambulatorial. A autora ainda destaca que os desdobramentos adversos da polifarmácia favorecem sinergismos e antagonismos não desejados, descumprimento das prescrições dos produtos clinicamente essenciais e gastos excedentes com os de uso supérfluo. Portanto, os critérios de Beers ${ }^{7}$ podem ser utilizados como guia para a adequada prescrição de medicamentos em idosos, uma vez que, aplicados, é possível reduzir iatrogenias, consultas e hospitalizações nesses pacientes, já que contribuem para prevenir o uso de MPI em idosos. ${ }^{31}$

Sabe-se, no entanto, que o aumento da utilização de medicamentos está associado ao risco da manifestação de eventos adversos aos medicamentos, como interações medicamentosas. ${ }^{20,32}$ Neste estudo, grande parte dos idosos que receberam algum MPI estava exposta a interações, o que merece atenção, devido às condições patológicas como insuficiência cardíaca, renal ou hepática, frequentes nestes pacientes, sendo determinantes da manifestação dos efeitos adversos dos medicamentos e interações. ${ }^{20,32}$
Foram identificados, conforme a classificação ATC, três grupos terapêuticos principais (tabela 5) envolvidos em interações medicamentosas. Destacaram-se os medicamentos usados no sistema cardiovascular, o que também foi verificado em estudos realizados com usuários de UBS de Pernambuco ${ }^{29}$ e São Paulo, ${ }^{20}$ sendo que os princípios ativos de maior risco foram digoxina, amiodarona, furosemida, captopril, propranolol e nifedipino. ${ }^{20}$

Neste contexto, quanto maior o número de medicamentos prescritos, maior o risco de o usuário estar recebendo um MPI e ainda estar exposto a interações medicamentosas. Assim, entende-se que seja fundamental avaliar os riscos e benefícios relacionados à farmacoterapia prescrita, que precisa ser monitorada e/ou ter suas dosagens reduzidas, pois muitas interações podem reduzir a efetividade dos medicamentos ou aumentar suas concentrações séricas - por exemplo, a digoxina, com potenciais riscos de toxicidade. ${ }^{11,12}$

Desta forma, a conscientização dosprescritores sobre as possíveis diminuições na capacidade funcional de idosos, as quais podem também estar relacionadas a certos medicamentos, é de suma importância, uma vez que a mensuração da qualidade de vida vem sendo incorporada à avaliação de resultados de atendimento à saúde. Assim, destacam-se as mudanças na mobilidade e riscos de quedas que podem ser precipitados por medicamentos, como aqueles que causam hipotensão ortostática, depressão do sistema nervoso central ou desordens de movimentos. ${ }^{32}$

Portanto, o conhecimento dos padrões de uso e de prescrição entre os idosos constitui uma medida indireta da ocorrência dos efeitos danosos, sendo o primeiro passo para se conhecer os riscos subjacentes à terapêutica farmacológica. Além disso, é preciso conhecer o perfil das reações adversas, dimensioná-las, identificar seus impactos clínicos, sociais e monetários. ${ }^{33}$ A prescrição de medicamentos para o paciente geriátrico representa um verdadeiro desafio, devido aos vários fatores peculiares deste grupo etário. Além 
disso, a farmacoterapia desses pacientes exige individualização cuidadosa, havendo necessidade de buscar equilíbrio entre os riscos da prescrição excessiva de fármacos desnecessários e as consequências negativas da subprescrição. 2,34

Diante do exposto, Oliveira \& Bárta ${ }^{35}$ sugerem que no ato da dispensação desses produtos sejam abordadas questões básicas visando promover um estilo de vida saudável, para prevenir ou tratar essas doenças, o que pode resultar, inclusive, na redução do uso de medicamentos, com melhora na qualidade de vida do usuário.

Nesse contexto, percebe-se que é de fundamental importância o trabalho em equipe, uma vez que a educação do paciente pode refletir sua conscientização quanto a seu estado de saúde e à necessidade do uso correto dos medicamentos, tornando o tratamento mais efetivo e seguro. ${ }^{24}$ Segundo os autores, a maior interação entre os profissionais de saúde, em especial o médico, o farmacêutico e o enfermeiro, pode reduzir a manifestação de eventos adversos aos medicamentos, reduzindo os custos do sistema de saúde. ${ }^{24}$

Os critérios de Beers são de grande relevância e podem ser utilizados em muitas instituições de saúde, desde que sejam constantemente revisados e atualizados. ${ }^{7}$ Uma vez que a perspectiva é um número cada vez maior de pessoas vivendo mais tempo, com maior risco de desenvolver múltiplas doenças crônicas e fazer uso de múltiplos fármacos por tempo prolongado, ${ }^{33}$ esses critérios são essenciais para a prescrição racional e específica para esta população.

Desta forma, racionalizar o uso de medicamentos e evitar os agravos advindos da polifarmácia, da prescrição errônea e da iatrogenia medicamentosa serão alguns dos grandes desafios relacionados à saúde pública do idoso. ${ }^{33}$ Sabe-se que o idoso exige uma terapêutica específica para suas particularidades, necessitando que os prescritores atentem para as influências que um fármaco pode exercer sobre outro, quando utilizados concomitantemente. É imprescindível o trabalho em equipe das diversas áreas da saúde, pois o idoso apresenta situações emocionais e de saúde que se inter-relacionam. ${ }^{27}$

Este estudo apresentou limitações: no que se refere à metodologia utilizada, a análise de prontuários e prescrições não permitiu conhecer as doenças ou comorbidades que justificassem a prescrição dos medicamentos identificados na pesquisa. Não foi possível, também, verificar o uso de medicamentos por meio da automedicação, o que poderia aumentar o número de MPI utilizados e a exposição a interações medicamentosas. Além disso, os dados obtidos se referem aos medicamentos prescritos, não sendo possível saber se realmente foram usados por esses idosos.

\section{CONCLUSÃO}

Com base nas prescrições analisadas, verificou-se que os idosos atendidos na UBS pesquisada receberam um elevado número de medicamentos, maior que os atendidos em outras UBSs e serviços de saúde em diferentes estados brasileiros. Tal fato requer atenção, devido às alterações características do envelhecimento e aos efeitos que poderão ser desencadeados. Além disso, parte desses idosos utiliza medicamentos potencialmente inapropriados, o que aumenta o risco de interações medicamentosas, podendo comprometer a segurança e a qualidade de vida desta população. Por outro lado, a polifarmácia e o uso de MPI podem estar sendo necessários, tendo em vista que foram identificados medicamentos indicados para as doenças crônicas prevalentes nesta faixa etária.

Diante disso, é importante realizar estudos que analisem criteriosa e individualmente a necessidade da prescrição e utilização de MPI, bem como a possibilidade de sua substituição. Quando seu uso for inevitável, deve-se instituir o acompanhamento do usuário, visando identificar a manifestação de efeitos indesejados e/ou interações medicamentosas.

Sugere-se, também, que sejam implementadas no serviços terapias não farmacológicas e ações 
preventivas, a fim de favorecer a redução no consumo de medicamentos e a utilização de recursos não farmacológicos para o manejo de problemas de saúde. Nesse contexto, destacase a importância de os profissionais da área da saúde estarem aptos a identificar a presença de alterações que ocorrem no organismo humano com o decorrer dos anos, sua influência no metabolismo e ação dos medicamentos, além dos reais riscos e benefícios da farmacoterapia disponível, o que favorecerá tanto a saúde dos idosos quanto os serviços existentes.

\section{REFERÊNCIAS}

1. Brasil. Ministério da Saúde, Secretaria de Atenção à Saúde, Departamento de Atenção Básica. Envelhecimento e saúde da pessoa idosa. Brasília: Ministério da Saúde, 2006.

2. Pereira SEM. Farmacoterapia geriátrica. In: SILVA, P. Farmacologia. $6^{\mathrm{a}}$ ed. Rio de Janeiro: Guanabara Koogan, 2002. p. 1220-5.

3. Coelho Filho JM, Marcopito LF, Castelo A. Perfil da utilização de medicamentos por idosos em área urbana do nordeste do Brasil. Rev Saúde Pública 2004;38(4):557-64.

4. Souza PM, Santos LL, Silveira CAN. Fármacos em idosos. In: Brasil. Ministério da Saúde, Secretaria da Ciência, Tecnologia e Insumos Estratégicos, Departamento De Assistência Farmacêutica e Insumos Estratégicos. Formulário Terapêutico Nacional 2008: Rename 2006. Brasília, DF: Ministério da Saúde, 2008. p. 26-9.

5. Locatelli J. Interações medicamentosas em idosos hospitalizados. Einstein (São Paulo) 2007;5(4):343-46.

6. Beers MH. Explicit criteria for determining potentially inappropriate medicationse by the elderly. An update. Arch of intern med 1997;157(14):1531-36.

7. Fick DM, Cooper JW, Wade WE, Walter JL, Maclean JR, Beers MH. Updating the Beers criteria for potentially inappropriate medication use in older adults: results of a US consensus panel of experts. Arch Intern Med 2003;163(22):2716-24.

8. Quinalha JV, Correr CJ. Instrumentos para avaliação da farmacoterapia do idoso: uma revisão. Rev Bras Geriatr Gerontol 2010;13(3):487-99.

9. Ribeiro AQ, Araújo CMC, Acurcio FA, Magalhães SMS, Chaimowcz. Qualidade do uso de medicamentos por idosos: uma revisão dos métodos de avaliação disponíveis. Ciênc Saúde Coletiva 2005;10(4):1037-45.

10. World Health Organization, Collaborating Centre for Drug Statistics Methodology. Anatomical Therapeutic Chemical ATC/DDD Index 2011[Internet]. Oslo: WHO; 2011 [acesso em 17 ago 2011]. Disponível em: http://www.whocc.no/atcddd/

11. Brasil. Ministério da Saúde, Secretaria de Ciência, Tecnologia e Insumos Estratégicos, Departamento de Assistência Farmacêutica e Insumos Estratégicos. Formulário terapêutico nacional 2010: Rename 2010. 2a. ed. Brasília, DF: Ministério da Saúde, 2010.

12. Baxter K; Rosário BA, Tradutora. Interações medicamentosas de Stockley: referência rápida. Porto Alegre: Artmed; 2010.

13. Soares MA, Fernández-Llimós F, Lança C, Cabrita J, Morais JA. Operacionalização para Portugal: critérios de Beers de medicamentos inapropriados nos doentes idosos. Acta Med Port 2008;2(5)1:441-52.

14. Loyola Filho AI, Uchoa E, Lima-Costa MF. Estudo epidemiológico de base populacional sobre uso de medicamentos entre idosos na Região Metropolitana de Belo Horizonte, Minas Gerais, Brasil. Cad Saúde Pública 2006;22(12):2657-67.

15. Oliveira CAP, Marin MJS, Marchioli M, Pizoletto BHM, dos Santos RV. Caracterização dos medicamentos prescritos aos idosos na Estratégia Saúde da família. Cad Saúde Pública 2009;25(5):1007-16.

16. Lucchetti M, Granero AL, Pires SL Gorzoni ML. Fatores associados à polifarmácia em idosos institucionalizados. Rev Bras Geriatr Gerontol 2010;13(1):51-8.

17. Faustino CG, Martins MA, Jacob-Filho W. Medicamentos potencialmente inapropriados prescritos a pacientes idosos ambulatoriais de clínica médica. Einstein (São Paulo) 2011;9(1 Pt 1):18-23.

18. Rozenfeld S. Prevalência, fatores associados e mau uso de medicamentos entre os idosos: uma revisão. Cad Saúde Pública 2003;19(3):717-24.

19. Pandolfi MB, Piazzolla LP, Louzada LL. Prevalência de polifarmácia em idosos residentes em instituição de longa permanência de Brasília, Distrito Federal. Brasília Méd 2010;47(1):53-8.

20. Simões MJS, Marques AC. Consumo de medicamentos por idosos segundo prescrição médica em Jaú-SP. Rev Ciênc Farm Básica Apl 2005;26(2):139-44. 
21. Araújo CMC, Magalhães SMS, Chaiamowicz F. Uso de medicamentos inadequados e polifarmácia entre idosos do programa saúde da família. Lat Am J Pharm 2010;29(2):178-84.

22. Acurcio FA, Silva AL, Ribeiro AQ, Rocha NP, Silveira MR, Klein $\mathrm{CH}$, et al. Complexidade do regime terapêutico prescrito para idosos. Rev Assoc Med Bras 2009;55(4):468-74.

23. Brasil. Ministério da Saúde, Secretaria de Políticas de Saúde, Departamento de Ações Programáticas Estratégicas. Plano de reorganização da atenção à hipertensão arterial e ao diabetes mellitus: hipertensão arterial e diabetes mellitus. Brasília: Ministério da Saúde, 2001.

24. Lyra Júnior DP, Amaral RT, Veiga EV, Cárnio EC, Nogueira MS, Pelá IR. A farmacoterapia no idoso: revisão sobre a abordagem multiprofissional no controle da Hipertensão Arterial Sistêmica. Rev Latinoam Enferm 2006;14(3):428-34.

25. Gomes HO, Caldas CP. Uso inapropriado de medicamentos pelo idoso: polifarmácia e seus efeitos. Ver Hosp Univ Pedro Ernesto 2008; 7(1)88-9.

26. Flores L M, Mengue SS. Uso de medicamentos por idosos em região do sul do Brasil. Rev Saúde Pública 2005;39(6):924-9.

27. Bueno CS, Oliveira KS, Berlezi EM, Eickhoff HM, Dallepiane LB, Girardon-Perlinei NMO, et al. Utilização de medicamentos e risco de interações medicamentosas em idosos atendidos pelo Programa de Atenção ao Idoso da Unijuí. Rev Ciên Farm Básica Apl 2009;30(3):331-8.
28. Brasil. Ministério da Saúde, Secretaria de Atenção à Saúde, Departamento de Atenção Básica. Hipertensão arterial sistêmica para o Sistema Único de Saúde. Brasília : Ministério da Saúde, 2006. (Cadernos de atenção Básica, 15) (Séria A. Normas e Manuais Técnicos).

29. Medeiros ACD, Costa AR, Palmeira AC, Simões MO, Caldeira CC. Utilização de medicamentos assistidos por uma farmácia comunitária. Lat Am J Pharm 2009;28(5):700-5.

30. Farfel JM, Accorsi TAD, Duenhas TA, Franken M, Doudement SP, Moran M, et al. Visitas à emergência relacionadas a efeitos adversos a drogas: o papel da prescrição inapropriada. Einstein (São Paulo) 2010;8(2 Pt 1):175-9.

31. Gorzoni ML, Fabbri RMA, Pires SL. Critérios de Beers-Fick e medicamentos genéricos no Brasil. Rev Assoc Med Bras 2008;54(4):353-6.

32. Bisson MP. Farmácia clínica e atenção farmacêutica. $2^{a}$ ed. Barueri, SP: Manole, 2007.

33. Oshima-Franco Y, Chorilli M, Bernardes ACA. Intoxicação medicamentosa no idoso. Saúde Rev 2005;7(15):53-1.

34. Flores LM, Colet CF. Riscos da polifarmácia em clientes idosos. In: Malagutti W, Bergo AMA, organizadores. Abordagem Interdisciplinar do idoso. Rio de Janeiro: Rubio, 2010. p. 291-302.

35. Oliveira KR, Bárta RL. Medicamentos dispensados pelo programa "Aqui tem Farmácia Popular" em uma drogaria no município de Panambi-RS. Contexto \& Saúde 2010;10(19):132-6. 